\title{
Giant Parotid Gland Liposarcoma
}

\author{
Elola $\mathrm{A}^{1 *}$, Sanou $\mathrm{SM}^{2}$, Nikièma $\mathrm{Z}^{3}$, Ouedraogo \\ $\mathrm{RWL}^{4}$ and Ouedraogo $\mathrm{AS}^{5}$ \\ ${ }^{1}$ Department of Surgery, University of Bobo Dioulasso, \\ Burkina Faso \\ ${ }^{2}$ Department of Surgery, ENT unit, Bobo Dioulasso, \\ Burkina Faso \\ ${ }^{3}$ Department of Radiology, University of Bobo Dioulasso, \\ Burkina Faso \\ ${ }^{4}$ Department of Surgery, ENT unit, University of Bobo \\ Dioulasso, Burkina Faso \\ ${ }^{5}$ Department of Anatomopathology, University of \\ Ouagadougou, Burkina Faso \\ *Corresponding author: Elola Abdoulaye, \\ Department of Surgery, ENT unit, University of Bobo \\ Dioulasso, Burkina Faso
}

Received: J une 07, 2017; Accepted: J une 28, 2017;

Published: July 05, 2017

\section{Introduction}

Liposarcoma is a rare type of cancer derived from primitive cells that undergo adipose differentiation [1]. Liposarcoma can appear in any part of the body, however it arises usually in the lower extremities, the retroperitoneum and the upper limbs [2,3]. The head and neck region represent only $1.8 \%$ to $6.2 \%$ of the cases $[4,5]$. Parotid liposarcoma is uncommon and is rather generally discovered at a relatively early stage.

The objective of this work was to describe the clinical characteristics of a case of parotid liposarcoma received at an advanced stage, and the management modalities of such cases in a developing country.

\section{Patient and Method}

We report a case of a giant parotid liposarcoma received in a 25 years old male man. We described the clinical particularities then we make a review of the literature of this infrequent disease.

\section{Case Presentation}

A young man 25 years old, without any particular pathologic antecedent was admitted in the ENT unit of the University Hospital Center Souro Sanou of Bobo Dioulasso, Burkina Faso, with a giant, ulcerated and hemorrhagic tumor of the left parotid gland.

The disease would have begun 7 months before, by a painless enlargement of the left parotid gland, gradually increasing. Four months after the beginning, the patient noticed the ulceration and bleeding of the tumor. In front of the persistence of repeated bleedings and the increase of the parotid mass, the patient is referred to the ENT unit of CHU Souro Sanou.

The examination noted a giant ulcerated, suppurated tumor of left parotid gland, approximately 15 X $8 \mathrm{~cm}$ (Figure 1), fixed, of firm consistency and bleeding at the contact.

Moreover there were bilateral cervical nodes and a light lockjaw.
The remainder ENT examination was normal. Required HIV serology returned negative.

CT scan showed (Figure 2) a giant expansive heterogeneous process $[A, B, C$, star], with areas of hypo attenuation $[A, B, C$, head of arrow] evoking lipoma, and micro calcifications [A, white arrow].

Moreover the presence of nodes under jawbones and neck [A, B, black arrow] were noted. In addition, a sagittal pulmonary view noted hyper density evoking pulmonary metastasis $[D$, white arrow].

The histological examination showed (Figure 3) tumor proliferation made of variable size cells presenting a lipogenic differentiation. The cells were equipped with pleomorphic, hyperchromatic nuclei with irregular borders. There were areas of tumour necrosis and pleomorphic lipoblasts.

Palliative care was instituted and consisted of administration of morphinic analgesics, antibiotics, iterative transfusions and local care. The evolution was marked by progressive general state deterioration, followed by the death of the patient.

\section{Discussion}

Liposarcomas are the most common soft tissue sarcomas in adults, but remain like all the sarcomas a rare disease. They derived from primitive cells that undergo adipose differentiation $[1,4]$ and do not rise from malignant transformation of lipomas [6].

They can appear in any part of the body. However they arise usually [2-5] in the thigh (more than $50 \%$ of cases), the retroperitoneum (30\% of cases), the upper limbs (10\% of cases). The head and neck region represent only $1.8 \%$ to $6.2 \%$ of the cases. The localizations in the parotid gland are very few reported. Only 15 cases reported in the literature since 1968 according to our Medline search.

Our patient was received 7 months after the beginning of the symptoms, at an advanced stage. The absence of pains at the 


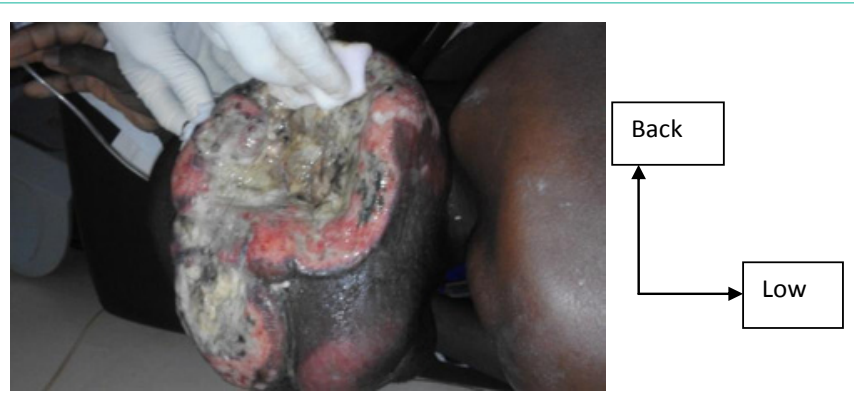

Figure 1: Giant left parotid gland liposarcoma ulcerated.

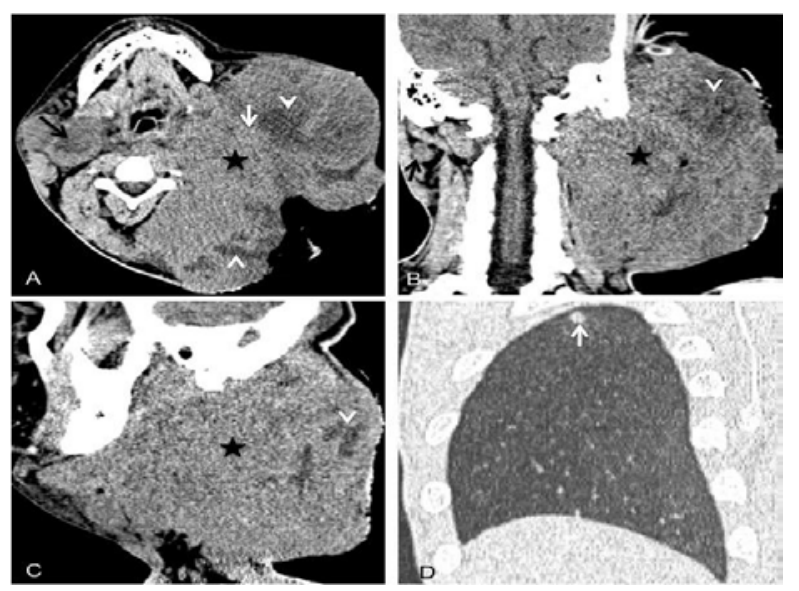

Figure 2: Cervical, cranial, encephalic and thorax CT scan obtained with and without injection of iodized contrast product.

A : Axial image without injection. B: Coronal image with injetion. C: Sagittal image with injection. D : Thorax image after injection.

beginning, the traditional care more often use in our context, and especially the high cost of modern care for the majority of the people can explain late consultations of our health centers by the patients.

Liposarcoma imaging is not specific however intra tumoral calcifications are rather evocative of sarcoma [2]. CT and Magnetic Resonance Imaging allow the assessment of extension.

The diagnosis of liposarcoma is based on the results of histological examination.

The last WHO classification of soft tissue tumours [7] distinguish well differenciated (low grade), dedifferentiated liposarcoma (high grade), myxoid (intermediaire grade), rounds cells (high grade), pleomorphic (high grade), liposarcoma not otherwise specified.

Metastasis can appear as our case in the lung, the liver and the bones [2].

The treatment of soft tissue sarcomas is based on radical excision of the tumor [2-4,6]. Sufficient margins are required: about $2 \mathrm{~cm}[7]$.

Our patient presented a giant hemorrhagic, unresectable pleomorphic liposarcoma of the left parotid gland.

Chemotherapy could have been managed if drugs were available and financially accessible, the patient and his family ensuring themselves care expenses. It would allow according to Gortzac [8] a substantial reduction of the size of the tumor. In January 2016, eribulin

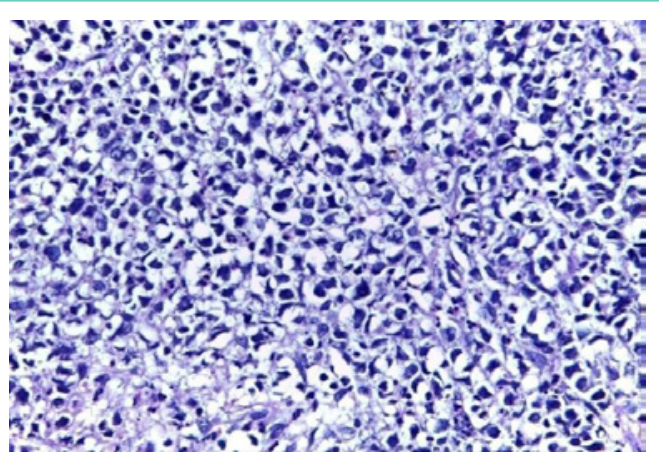

Figure 3: Pleomorphic liposarcoma. Diffuse cell proliferation constituted of cells presenting lipogenic differentiation with important atypies.

(Halaven) was approved by the US Food and Drug Administration [9] for unresectable or metastatic liposarcoma. The objective of radiotherapy would be tumoral loco regional control [10].

The pronostic evaluation of liposarcoma is based on the grade, the size and the localization of the tumor, the quality of initial surgery, the presence of metastasis or not.

According to these items our patient who had a giant metastatic high grade and unresectable tumor had a very bad prognostic.

\section{Conclusion}

This case underlines the recurrent problem of late consultations in developing countries. In the same way, the availability and the financial accessibility of chemotherapy remain a whole problem. So, for us the implementation of sickness insurance must be a priority in our countries.

\section{References}

1. Huh WW, Yuen C, Munsell M, Hayes-Jordan A, Lazar AJ, Patel S, et al. Liposarcoma in Children and Young Adults: A Multi-Institutional Experience. Pediatr Blood Cancer. 2011; 57: 1142-1146.

2. Julieron M, Robin YM, Penel N, Chevalier D. Sarcomes de la tête et du cou. EMC - Oto - rhino - laryngologie. 2013; 8: 1-19.

3. Galy-Nernadoy C, Garrel R. Sarcomes des tissus mous de la tête et du cou de l'adulte. Annales françaises d'oto-rhino-laryngologie et de pathologie cervico-faciale. 2016; 133: 34-39.

4. Chakraborty S, Kassel EE, Yu E. Nasopharyngeal liposarcoma MRI imaging features and a review of the literature. The British journal of radiology. 2007; 80: $145-146$.

5. Papacharalampous GX, Kikidis D, Vasileiou A, Bousiotou A, Chrysovergis A. Liposarcoma of the Nasopharynx: Diagnosis and Management of a Rare Diagnostic Entity.

6. Gebhardt M, Buecker PJ, Traduit en français par Baconnier S, Espesset E. Les liposarcomes [En ligne] Disponible sur.

7. Von Mehren M, Randall RL, Benjamin RS, Boles S, Bui MM. NCCN clinica practice guidelines in oncology [NCCN Guidelines] soft tissue sarcoma. J Natl Compr Canc Netw. 2014; 12: 473-483.

8. Gortzak E, Azzarelli A, Buesa J, Bramwell VH, van Coevorden F, van Geel AN, et al. EORTC. Soft tissue bone sarcoma group and the National Cancer Institute of Canada Clinical Trials Group/ Canadian Sarcoma Group. A randomised phase II study on neoadjuvant chemotherapy for "high risk" adult soft tissue sarcoma [archive], Eur J Cancer. 2001; 37: 1096-1103.

9. Robert A Schwartz. Liposarcoma Treatment \& Management.

10. Tran LM, Mark R, Meier R. Sarcoma of the head and neck. Prognostic factors and treatment strategies. Cancer. 1992; 70: 169-177. 